\title{
Consumer-Data Approach to Assess the Effect of Residential Grid-Tied Photovoltaic Systems and Electric Vehicles on Distribution Transformers
}

\author{
Fabian M. Uriarte, Amir Toliyat, Student Member, IEEE, Alexis Kwasinski, Member, IEEE, and \\ Robert E. Hebner, Fellow, IEEE
}

\begin{abstract}
The authors examine the impact of residential photovoltaic arrays and electric vehicles on distribution transformers by using 3-D surface and 2-D filled contour plots. These visualizations, somewhat unorthodox to power distribution analysis, elucidate the impact of hundreds of assets on distribution transformers on a single view. The visualizations are created with a smart grid computer model that accepts residential electrical recordings in one minute intervals. An analysis of simulation results shows that the electrical footprint experienced by a residential community and its distribution transformers stems from photovoltaic arrays rather than from electric vehicles. Additionally, the results indicate the existing distribution assets may be ready to support the proliferation of photovoltaic arrays and electric vehicles, a common concern across utilities in the United States.
\end{abstract}

Index Terms-Cloud, Distribution, Electric, Grid, Model, Power, Simulation, Solar, Smart, Transformer, Utility, Vehicle

\section{INTRODUCTION}

$\mathrm{T}_{\mathrm{s}}^{\mathrm{h}}$ growth of household grid-tied photovoltaic (PV) systems is stimulating the need for studies of the impact of this technology on electrical distribution systems [1]. The effects of the growth of this renewable and intermittent source can range from changes in voltage profiles [2], to impact on transformer life, to significant changes in power factor.

The conventional approach to this assessment is to combine load flow with selected dynamic analysis to address various scenarios of intermittency [1,3]. This paper shows that another approach is becoming possible with smart metering. This approach is to combine actual recorded data from individual field-metered sources and loads with a computer model of the distribution system to understand how solar intermittency and load variations affect the distribution system. Fundamentally, this approach takes advantage of the additional timely data available about the distribution system to gain an improved insight into the system from the substation to the loads.

Privacy and other concerns make it infeasible at this time to use the smart meter data directly for distribution system studies. Therefore, to highlight some of the attributes of the approach, the data used was volunteered to Pecan Street, Inc. by the residents of the Mueller subdivision in Austin, Texas.

The authors are with the Center for Electromechanics of The University of Texas at Austin. (e-mails: \{f.uriarte, r.hebner\}@cem.utexas.edu, \{atoliyat, akwasins\}@mail.utexas.edu). Pecan Street, Inc. supported this project via a grant from the Department of Energy.
The data was rendered anonymous by a third party and stored in the supercomputer facility at the University of Texas at Austin.

Pecan Street, Inc. is a non-profit research and commercialization organization headquartered at the University of Texas at Austin (UT). This organization is a significant creator of original customer energy use and behavioral research data, and operates data-intensive field trials open to researchers and member companies. Pecan Street's core research assets include research data on consumer electricity and natural gas use.

This paper analyzes the impact of customer-owned residential grid-tied photovoltaic arrays (PVs) and electric vehicles (EVs) on utility-owned pad-mounted distribution transformers. The data provided by Pecan Street, Inc. is used in conjunction with a computer model of a residential community to estimate the system's state. This novel analysis approach addresses the positive and negative impacts of concentrations of residential PVs and EVs. In contrast to orthodox modeling techniques, this work uses real electrical data recorded from hundreds of homes in one-minute intervals. Real data promotes confidence in the results, as it accounts for the effects of un-correlated load behavior and intermittent weather conditions pertinent to the geographical area under consideration.

\section{The Mueller COMMUNITY SMART GRID}

The Mueller development is a 711-acre mixed use development in Austin, Texas, in which every new building is green built (as certified through LEED or Austin Energy's nationally recognized Green Building program). This development is an urban in-fill redevelopment built on the site of Austin's former municipal airport (closed in 1999). The Mueller Community is a prototypical development model, similar to hundreds like it across the United States.

Nearly 200 homes at Mueller are instrumented with secondary (second energy meter) and tertiary (inside homes) meters to report electrical data in one-minute and one-second intervals. Many homes are equipped with PV systems, of which $40 \%$ face west (the nation's highest residential concentration of west-facing, load-aligned PV generation). A picture showing this high concentration of PVs is shown in Fig. 1. 


\section{ELECTRIC SERVICE}

A one-line diagram of the Mueller community is shown in Fig. 2. Each substation transformer serves three feeders at $12.47 \mathrm{Y} / 7.2 \mathrm{kV}$. Each feeder, in turn, serves other urban communities and commercial areas via service laterals in addition to the Mueller community. Shown from left to right is the lateral service entrance to the Mueller community. The thicker lines in Fig. 2 represent three-phase overhead lines or underground cables. The thin lines inside the community (in corresponding color) represent single-phase distribution cables. Single-phase cables run from switchgear boxes (boxes 1,2 , and 3) to each distribution transformer in a circuit. Each phase incoming from the lateral to switchgear box 1 is split into sub-circuits. For example, phase $b$ (at switchgear box 1 ) splits into circuits B2 and B4. These circuits operate in openloop configuration, as indicated by the dashed-line segments.

Each circuit is protected by 65 A fuses at the switchgear boxes, and provides electric service to its downstream transformers. The primary-side service voltage to each transformer (7.2 kV, phase to ground) is stepped down to a usage level of 240/120V (split-phase). The distribution transformer types are tabulated in Table I along with a summary of the PV systems and EVs assigned to each transformer. Each transformer serves several homes and several EVs. Some transformers have PVs behind them, others do not. The asset distribution and count considered herein constitutes the existing infrastructure at the Mueller community today. The asset count for each transformer is tabulated in Tables II through IV.

\section{SimULATION RESULTS AND ANALYSIS}

The initial investigation used actual measured load data and an electrical circuit model to assess the effect that the existing circuit topology and the distribution of sources and loads had on distribution transformers. The results were obtained by using collected data provided by Pecan Street, Inc. (Feb. 2012) in one-minute intervals in conjunction with a computer model built in MATLAB/Simulink. The collected data was used as load shapes (i.e., $\mathrm{kW}$ vs. time) for the residential consumption and as generation profiles for the rooftop PV systems. The computer model accepts these load shapes and estimates voltage, current, power, power factor, and energy usage everywhere else in the network.

\section{A. Residential Load}

Figure 3 shows the power consumption profiles of each of the 735 homes. These profiles exclude electric vehicle

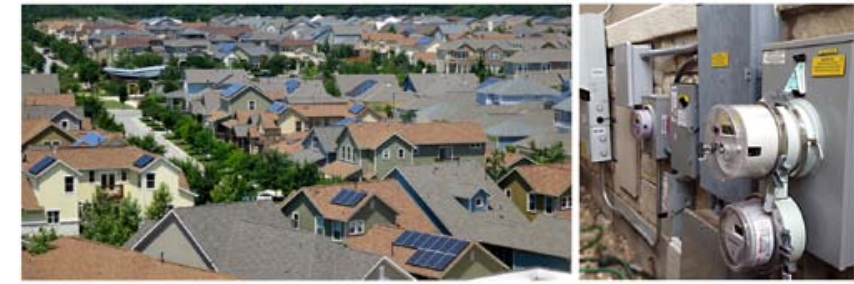

Fig. 1. Left: Mueller homes equipped with rooftop PVs (6 kW each). Right: Second (research) meter in a dual socket configuration. (Image source: Pecan Street, Inc.) charging and PV generation. Explanations of each view in the figure are as follows:

a) Figure 3 (a) shows the active power consumption (kW) of each of the homes over a 24-hour period. The data is graphed using a mesh grid to produce a 3-D surface plot. The color map corresponding to this surface appears on the right side of the figure, where power consumptions $>10 \mathrm{~kW}$ appear in increasingly-dark red color. The advantage of this view is that it permits detecting correlation in consumption behavior by observing the peaks and the valleys on the surface. For example, consider the circled areas of this view. Each area encloses a different group of homes at similar times of the day ( 12 PM through 12 AM). The top circle encloses several residences exceeding $10 \mathrm{~kW}$ (simultaneously) while the lower circle encompasses other residences going through consumption valley below $4 \mathrm{~kW}$ (during the same times of the day). The data highlights the fact that residential loads are heterogeneous and their individual behavior is difficult to predict.

b) Figure 3 (b) exhibits load-level duration using a contour plot. For example, referring to the circled areas corresponding to Fig. 3 (a), the $10 \mathrm{~kW}$ peaks only last a few minutes. Similarly, the lower circled area, in this view, shows that the valleys of $\sim 4-5 \mathrm{~kW}$ last much longer than the $8-12 \mathrm{~kW}$ peaks do. Additionally, not all homes incur such peaks, only some do, which is clearly noticeable from this type of view. Also,

TABLE I

DISTRIBUTION TRANSFORMER TYPES

\begin{tabular}{rccrrrr}
\hline kVA & $\begin{array}{c}\text { Primary } \\
\text { Voltage } \\
\text { (V) }\end{array}$ & $\begin{array}{c}\text { Secondary } \\
\text { Voltage } \\
\text { (V) }\end{array}$ & $\begin{array}{c}\text { No load } \\
\text { losses } \\
\text { (W) }\end{array}$ & $\begin{array}{c}\text { Loaded } \\
\text { losses } \\
\text { (W) }\end{array}$ & Z \% Count \\
\hline 25 & 7,200 & $240 / 120$ & 71 & 228 & 2.3 & 6 \\
50 & 7,200 & $240 / 120$ & 105 & 404 & 2.3 & 74 \\
75 & 7,200 & $240 / 120$ & 167 & 456 & 2.5 & 11 \\
100 & 7,200 & $240 / 120$ & 181 & 683 & 2.5 & 2 \\
167 & 7,200 & $240 / 120$ & 248 & 1234 & 3.0 & 1 \\
\hline & & & & & Total & 94 \\
\hline
\end{tabular}

TABLE II

ASSET COUNT FOR EACH DISTRIBUTION TRANSFORMER IN PHASE A

\begin{tabular}{ccccrrr}
\hline $\begin{array}{c}\text { Phase \& } \\
\text { Circuit }\end{array}$ & \multirow{2}{*}{ Xfm. \# } & Xfm. ID & kVA & \multicolumn{3}{c}{ \# Homes \# PVs \# EVs } \\
\hline A1 & 1 & T01A1,50 & 50 & 8 & 1 & 0 \\
A1 & 2 & T02A1,50 & 50 & 10 & 1 & 0 \\
A1 & 3 & T03A1,50 & 50 & 10 & 4 & 2 \\
A1 & 4 & T04A1,50 & 50 & 8 & 3 & 2 \\
A1 & 5 & T05A1,75 & 75 & 11 & 0 & 1 \\
A1 & 6 & T06A1,50 & 50 & 8 & 3 & 2 \\
A1 & 7 & T07A1,50 & 50 & 8 & 2 & 2 \\
A1 & 8 & T08A1,50 & 50 & 10 & 4 & 4 \\
A1 & 9 & T09A1,50 & 50 & 8 & 3 & 2 \\
A1 & 10 & T10A1,50 & 50 & 8 & 2 & 2 \\
A1 & 11 & T11A1,50 & 50 & 9 & 4 & 2 \\
A1 & 12 & T12A1,50 & 50 & 8 & 2 & 0 \\
A1 & 13 & T13A1,50 & 50 & 8 & 2 & 0 \\
A1 & 14 & T14A1,50 & 50 & 9 & 5 & 4 \\
A1 & 15 & T15A1,50 & 50 & 10 & 5 & 2 \\
A1 & 16 & T16A1,50 & 50 & 10 & 1 & 1 \\
A1 & 17 & T17A1,50 & 50 & 7 & 1 & 0 \\
A1 & 18 & T18A1,50 & 50 & 10 & 5 & 1 \\
A1 & 19 & T19A1,50 & 50 & 8 & 6 & 2 \\
A1 & 20 & T20A1,50 & 50 & 10 & 4 & 3 \\
A1 & 21 & T21A1,50 & 50 & 8 & 2 & 1 \\
\hline & & & Totals & 186 & 60 & 33 \\
\hline
\end{tabular}


TABLE IIII

ASSET COUNT FOR EACH DISTRIBUTION TRANSFORMER IN PHASE B

\begin{tabular}{|c|c|c|c|c|c|c|}
\hline $\begin{array}{c}\text { Phase \& } \\
\text { Circuit }\end{array}$ & Xfm. \# & Xfm. ID & kVA & \# Homes & \# PVs \# & \# EVs \\
\hline B2 & 22 & T22B2,25 & 25 & 3 & 0 & \\
\hline B2 & 23 & Т23B2,50 & 50 & 7 & 0 & \\
\hline B2 & 24 & T24B2,75 & 75 & 11 & 4 & \\
\hline B2 & 25 & T25B2,50 & 50 & 7 & 3 & \\
\hline B2 & 26 & T26B2,50 & 50 & 8 & 3 & \\
\hline B2 & 27 & T27B2,50 & 50 & 8 & 5 & \\
\hline B2 & 28 & T28B2,50 & 50 & 7 & 1 & \\
\hline B2 & 29 & Т29B2,50 & 50 & 7 & 5 & \\
\hline B2 & 30 & Т30В2,50 & 50 & 7 & 4 & \\
\hline B2 & 31 & T31B2,50 & 50 & 10 & 6 & \\
\hline B2 & 32 & Т32В2,50 & 50 & 8 & 4 & \\
\hline B2 & 33 & Т33В2,50 & 50 & 8 & 1 & \\
\hline B2 & 34 & Т34B2,50 & 50 & 8 & 1 & \\
\hline B2 & 35 & Т35В2,50 & 50 & 5 & 1 & \\
\hline B2 & 36 & Т36В2,50 & 50 & 4 & 1 & 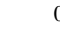 \\
\hline B2 & 37 & Т37B2,100 & 100 & 10 & 1 & c \\
\hline \multirow[t]{2}{*}{ B2 } & 38 & Т38В2,75 & 75 & 9 & 2 & 2 \\
\hline & & & Totals & 127 & 42 & 31 \\
\hline
\end{tabular}

\begin{tabular}{crrrrrr}
\hline $\begin{array}{c}\text { Phase \& } \\
\text { Circuit }\end{array}$ & Xfm. \# & Xfm. ID & \multirow{2}{*}{ kVA } & \# Homes \# PVs \# EVs \\
\hline B4 & 39 & T61B4,50 & 50 & 7 & 1 & 1 \\
B4 & 40 & T62B4,50 & 50 & 7 & 1 & 0 \\
B4 & 41 & T63B4,50 & 50 & 6 & 0 & 0 \\
B4 & 42 & T64B4,50 & 50 & 10 & 1 & 1 \\
B4 & 43 & T65B4,50 & 50 & 8 & 0 & 1 \\
B4 & 44 & T66B4,50 & 50 & 6 & 1 & 0 \\
B4 & 45 & T67B4,50 & 50 & 6 & 1 & 0 \\
B4 & 46 & T68B4,50 & 50 & 6 & 3 & 1 \\
B4 & 47 & T69B4,50 & 50 & 7 & 2 & 2 \\
B4 & 48 & T70B4,50 & 50 & 8 & 5 & 1 \\
B4 & 49 & T71B4,25 & 25 & 5 & 2 & 2 \\
B4 & 50 & T72B4,25 & 25 & 4 & 2 & 1 \\
B4 & 51 & T73B4,50 & 50 & 4 & 2 & 0 \\
B4 & 52 & T74B4,50 & 50 & 7 & 3 & 2 \\
B4 & 53 & T75B4,50 & 50 & 10 & 3 & 2 \\
B4 & 54 & T76B4,25 & 25 & 4 & 0 & 0 \\
B4 & 55 & T77B4,25 & 25 & 5 & 2 & 0 \\
B4 & 56 & T78B4,25 & 25 & 1 & 0 & 0 \\
B4 & 57 & T79B4,50 & 50 & 10 & 3 & 0 \\
B4 & 58 & T80B4,50 & 50 & 10 & 3 & 1 \\
B4 & 59 & T81B4,50 & 50 & 10 & 3 & 3 \\
B4 & 60 & T82B4,50 & 50 & 10 & 3 & 1 \\
B4 & 61 & T83B4,50 & 50 & 10 & 1 & 1 \\
B4 & 62 & T84B4,50 & 50 & 8 & 1 & 1 \\
B4 & 63 & T85B4,50 & 50 & 10 & 0 & 0 \\
B4 & 64 & T86B4,75 & 75 & 10 & 0 & 0 \\
B4 & 65 & T87B4,50 & 50 & 6 & 1 & 1 \\
B4 & 66 & T88B4,75 & 75 & 11 & 0 & 1 \\
\hline & & & Totals & 206 & 44 & 23 \\
\hline
\end{tabular}

note that the dominant color on this contour plot is dark blue. This color suggests that, most of the time, the residential consumption is near $2 \mathrm{~kW}$.

c) Figure 3 (c) shows the home consumptions as seen from the time-kW plane. Although it is expected that residential load behaves in unison in the evening hours, this view shows 735 load shapes, which suggests there is little correlation in residential load patterns. As it can be observed, the peaks and valleys of different homes are not necessarily aligned. Each home behaves independently of the others, with peak power consumptions occurring in the middle of the day, in the evening, or not occurring at all. This behavior is desirable for electric utilities because transformer loading tends to be closer to an average than the sum of the peak demands. This shows that any pricing or other signals that tend to correlate demand
TABLE IV

ASSET COUNT FOR EACH DISTRIBUTION TRANSFORMER IN PHASE C

\begin{tabular}{|c|c|c|c|c|c|c|c|}
\hline $\begin{array}{c}\text { Phase \& } \\
\text { Circuit }\end{array}$ & Xfm. \# & Xfm. ID & kVA & \# Homes & \# PVs F & $\# \mathrm{EV}$ & \\
\hline C3 & 67 & Т39С3,75 & 75 & 9 & 1 & & \\
\hline C3 & 68 & T40C3,75 & 75 & 11 & 0 & & \\
\hline C3 & 69 & T41C3,50 & 50 & 7 & 5 & & \\
\hline C3 & 70 & T42C3,50 & 50 & 10 & 3 & & \\
\hline C3 & 71 & Т43С3,50 & 50 & 8 & 1 & & \\
\hline C3 & 72 & T44C3,75 & 75 & 12 & 1 & & \\
\hline C3 & 73 & T45C3,50 & 50 & 2 & 0 & & \\
\hline C3 & 74 & T46C3,50 & 50 & 11 & 1 & & \\
\hline C3 & 75 & T47C3,50 & 50 & 5 & 4 & & \\
\hline C3 & 76 & T48C3,50 & 50 & 7 & 1 & & \\
\hline C3 & 77 & T49C3,50 & 50 & 4 & 0 & & \\
\hline C3 & 78 & T50C 3,100 & 100 & 11 & 2 & & \\
\hline C3 & 79 & T51C3,75 & 75 & 4 & 0 & & \\
\hline C3 & 80 & T52C3,50 & 50 & 3 & 0 & & \\
\hline C3 & 81 & Т53С3,167 & 167 & 8 & 2 & & \\
\hline C3 & 82 & T54C3,50 & 50 & 10 & 2 & & 1 \\
\hline C3 & 83 & T55C3,50 & 50 & 10 & 3 & & \\
\hline C3 & 84 & T56C3,75 & 75 & 7 & 1 & & \\
\hline C3 & 85 & T57C3,50 & 50 & 8 & 1 & & 1 \\
\hline C3 & 86 & T58C3,50 & 50 & 7 & 1 & & 1 \\
\hline C3 & 87 & T59C3,50 & 50 & 5 & 1 & & 1 \\
\hline \multirow[t]{2}{*}{ C3 } & 88 & T60C 3,50 & 50 & 6 & 1 & & 1 \\
\hline & \multicolumn{3}{|r|}{ Totals } & 165 & 31 & & 16 \\
\hline \multicolumn{8}{|l|}{$\begin{array}{c}\text { Phase \& } \\
\text { Circuit }\end{array}$} \\
\hline C5 & 89 & T89C5,75 & 75 & 10 & 0 & & 2 \\
\hline C5 & 90 & T90C5,50 & 50 & 8 & 0 & & 1 \\
\hline C5 & 91 & Т91C5,50 & 50 & 8 & 0 & & 0 \\
\hline C5 & 92 & T92C5,50 & 50 & 9 & 1 & & 0 \\
\hline C5 & 93 & T93C5,50 & 50 & 8 & 0 & & 0 \\
\hline \multirow[t]{2}{*}{$\mathrm{C} 5$} & 94 & T94C5,50 & 50 & 8 & 0 & & 0 \\
\hline & & & Totals & 51 & 1 & & \\
\hline
\end{tabular}

may result in an adverse effect on transformer life, because it stimulates longer operating times at higher temperatures [4]. It is also interesting to note that the average consumption over 24 hours for the 735 homes (computed in time intervals of one minute) is $0.95 \mathrm{~kW}$. This value was computed from actual recorded data in one minute intervals, which raises confidence in the result. However, this average value varies by day of the week, time of the year, and is not general.

d) Figure 3 (d) indicates the aggregated (sum) power consumption of all homes and approximates the total demand (excluding EVs, PV systems, and distribution power losses) as seen from the lateral service entrance. The power factor, which refers to displacement power factor rather than total power factor [5], is plotted against the right-side axis. This power factor (an estimated aggregate result based on the estimated power factor of each home varying between 0.7 lagging and 0.7 leading.) is observed at the lateral level, not at the individual home level. As noticed from the real and total power traces, the peak power demand occurs in the evening (as expected). In contrast to Fig. 3 (c), which is shown at the individual home level, at the lateral level the power demand shape is repeatable, predictable and interestingly, results from averaging mostly unpredictable and uncorrelated home consumption profiles. As recognized from the peak total power, the peak load of this community is 1.5 MVA, but varies by day of the week and time of the year.

\section{B. PV Generation}

Figure 4 shows the generation profiles of each roofmounted, residential PV arrays. These grid-tied residential PV systems only produce real power [6, 7], and not reactive power [8],[9]. This contributes to low power factors as seen from the 


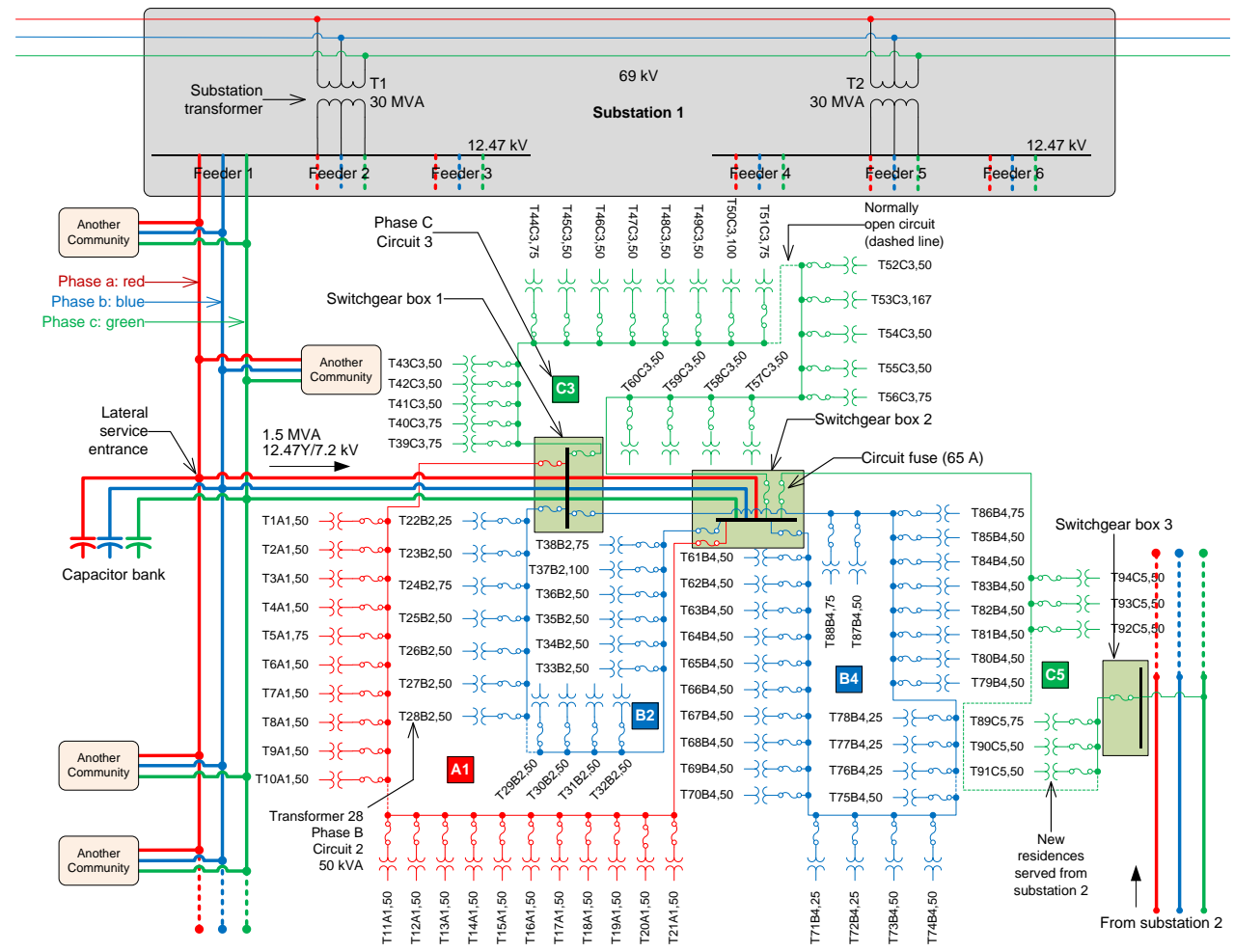

Fig. 2. One-line diagram of Mueller Community including 735 homes, 94 transformers, 176 PVs, and 100 EVs (Austin, Texas)

lateral service entrance. The meaning of each view of the PV generation views is explained next:

a) Figure 4 (a) shows the individual real power generation output of all PVs as a 3-D surface. The color map corresponding to this surface appears on the right side of the figure, where generation $>4.5 \mathrm{~kW}$ is indicated in increasingdark red. This view clearly shows that the peak generation of each residential PV system can be similar, but is not the same. Also, it is apparent that the envelope of this surface follows a pattern similar to a normal (Gaussian) distribution curve. The circled area shows a group of PV systems with irradiance deficiency. Various PV system characteristics, such as rated power, number of cells, connection method, dimensions, cell technology, irradiance based on sun's azimuth and zenith angles, system efficiency, dynamic variations in atmospheric and environmental air mass, passing clouds inducing whole or scattered shaded, temperature, inverter control and circuit topology, and maximum power point tracking approach, all affect PV power output. Hence, the effect of PV sources on the grid depends on these and other PV system characteristics. Evidently, due to the geographical separation of the PV systems, at a given instant equal PV systems may not be affected by these characteristics in the same way. In particular, unequal irradiance geographical distribution highlights an important fact of distributed generation, not all PV systems are affected simultaneously by cloud events; many PV systems retain high power outputs, while others do not. This lack of correlation among the distributed PV system outputs is advantageous in terms of voltage stability [10] when compared to concentrated PV plants [9, 11, 12].

b) Figure 4 (b) shows data in Fig. 4 (a) as seen from the homehour plane. The encircled areas show the time when some PV systems receive low irradiance levels (from 6 to $11 \mathrm{AM}$ ). This view also helps in counting the number of PVs affected (PV $\# 120$ through \#140). Another advantage of this view is that it permits comparing the generation profiles of south-facing and west-facing PV arrays. The homes at Mueller have west-, south-, or a combination of both PV orientations. The advantage of dual orientations is a wider time-coverage including better alignment with the evening load. From the color map on the right, the predominant output power color of the PV systems is closer to $4 \mathrm{~kW}$ than it is to $5 \mathrm{~kW}$. This indicates that PV systems spend more time producing power at shoulder output levels rather than at their peak output.

c) Figure 4 (c) shows that residential grid-tied PV systems are correlated in the coarse sense (not in a detailed sense). Cloud coverage may or may not reduce the output of all PV arrays. If PV systems were more distributed, their output would be reduced sequentially from one to the next, but these results are not general. The PV systems depend on cloud type, height, time of day, season, and the factors mentioned for Fig. 4 (a), which lie outside the scope of this study. An advantage of having recorded PV data is that intermittent irradiance and solar ramp rates [13] need not be modeled; these effects are part of the measured data and are injected into the computer model using the generation profile of each PV system.

d) Figure 4 (d) shows aggregated residential PV power generation. Although the individual PV power generation is intermittent and uncorrelated in the short term, at the aggregate level, the generation follows a predictable smooth envelope that reaches nearly $0.5 \mathrm{MW}$ per day. The aggregated power generation, however, is less than the total residential load shown in Fig. 3 (d). This result indicates, while this community has a high density of PV generation, there is no excess PV power that leaves the community through the lateral 


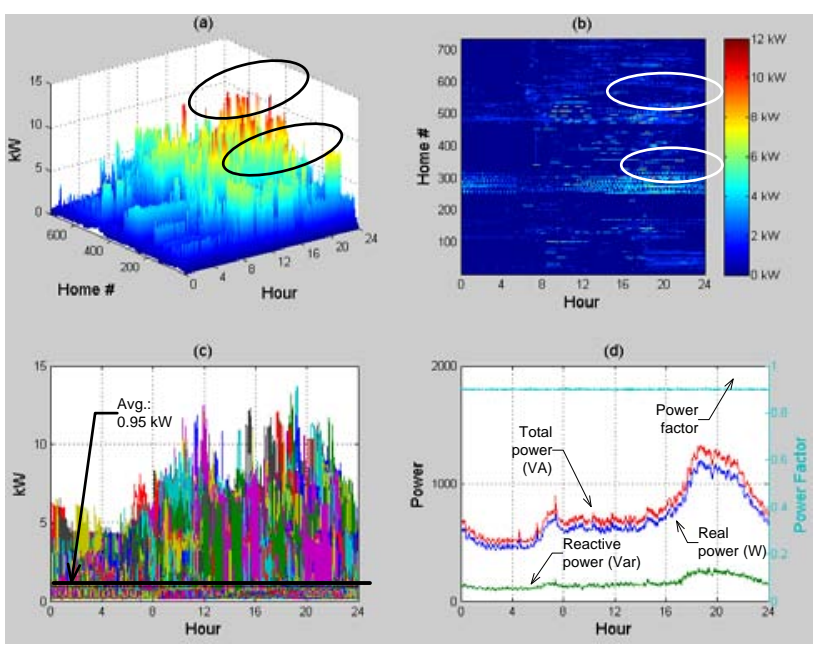

Fig. 3. Residential consumption for all homes in a 24-hour period. Data interval: $1 \mathrm{~min}$.

service entrance. Instead, the excess PV power generation behind the distribution transformers is consumed as billable energy ( $\mathrm{kWh}$ ) by other homes on the same electrical phase. (It is also noted that the utility provides credit towards a residential customer's bill for any power not consumed locally.)

\section{Electric Vehicles}

Figure 5 shows the charging profiles for 100 plug-in hybrid EVs. The charging profiles include a realistic mix of 120 and 240 V charging profiles (as reported by Pecan Street, Inc.). The following analysis is an example of using the model to project the effect of changes in load. Although actual charging profiles were recorded for a Chevy Volt (which charges at a lower $\mathrm{kW}$ than is now available in other electric vehicles), the projected number of electric vehicles have not yet been purchased or leased. To predict the effect of the full set, the 100 vehicles were assigned to the homes of consumers expecting to acquire an electric vehicle. In addition, the state of charge at the initiation of the charging process was randomized and the starting time for a charge was randomized between 4 and 8 PM.

a) Figure 5 (a) demonstrates the load consumption of each EV using a surface plot. The curve peaks indicate groups of vehicles charging at $240 \mathrm{~V}$. Lower peaks originate in EVs charging at $120 \mathrm{~V}$. The mix of high and low peaks is a direct result of randomizing charge profile levels. The vehicles charging at $240 \mathrm{~V}$ appear in red, which corresponds to charging levels of $3.3 \mathrm{~kW}$. The lower peaks corresponding to $120 \mathrm{~V}$ appear in cyan and correspond to a charging level of $1.44 \mathrm{~kW}$. There is also a set of vehicles that charge at 0.96 $\mathrm{kW}$, and appear as light blue peaks. As aforementioned, the plug-in times were randomized to reflect a typical behavior in a residential neighborhood. The initiating charging time was constrained between 4-8 PM for two reasons. First, to highlight any evening charging problems and assess the potential impact these charging profiles may have on the distribution transformers. Second, plug-in times after 4 PM reduce electrical overlap with PV systems.

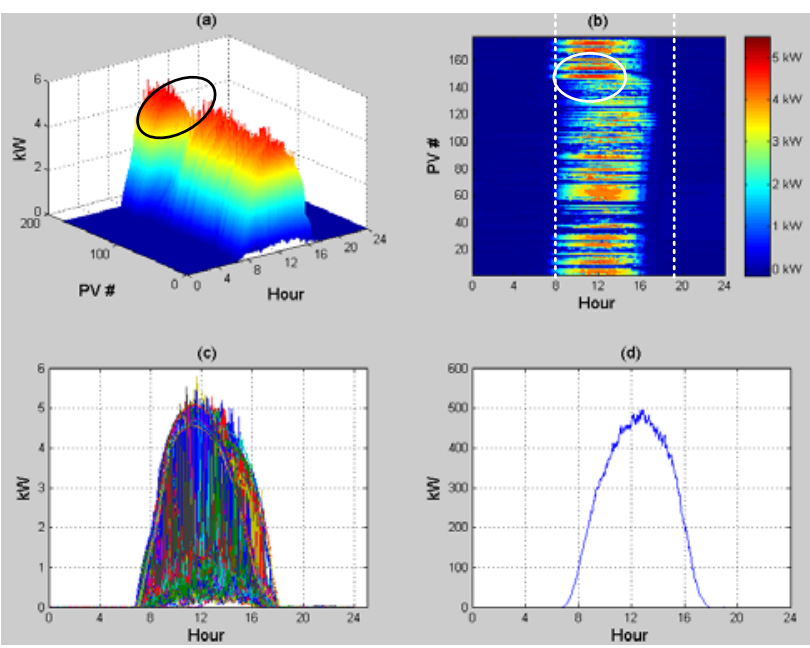

Fig. 4. Solar generation for all $178 \mathrm{PVs}$ in a 24-hour period. Data interval: 1 min..

b) Figure 5 (b) highlights the assumed EV charging characteristics by showing charge time spans. These time spans indicate how long each vehicle charges from a random level of state-of-charge at plug-in time. Some vehicles charge overnight and into the early morning as shown by the circled area. These vehicles charge at $120 \mathrm{~V}$, consuming $0.96 \mathrm{~kW}$ or $1.44 \mathrm{~kW}$. The short time spans shown in this view represent both $240 \mathrm{~V}$ charging $\$ 3.5$ hours) and $120 \mathrm{~V}$ charging ( $\leq 9$ hours). In the $120 \mathrm{~V}$ case, short time spans represent vehicles with a higher state-of-charge at plug-in time.

c) Figure 5 (c) shows some un-correlation in the EV charging, but not as much the residential consumption or PV generation since the EV charging plug-in time was set between 4-8 PM. Uncorrelated charging is desirable from the utility stand-point as it reduces the duration of peak power and therefore peak temperature experienced by the transformer. Transformer life degrades more rapidly at higher operating temperatures. However, the benefit of uncorrelated power demand is not new; it has been observed for decades in residential airconditioner usage (e.g., many units operate in unison while the operation of many other units is un-correlated. The time spans in this view are further annotated with labels showing the three possible charging levels for the vehicles. (The time in hours indicates the maximum possible charging duration, not the actual time a vehicle charged for.) It is noted that $240 \mathrm{~V}$ charging requires level-2 chargers with a dedicated $240 \mathrm{~V}$ circuit [14] inside a residence.

d) Figure 5 (d) exemplifies the total EV charging load at the lateral level based on the assumed charging profile. The largest EV impact based on EV charging power consumption occurs between 6-8 PM and appears to be $<200 \mathrm{~kW}$ on a typical day. This result is strongly dependent on the charging profiles assumptions and absent incentives that drive a higher degree of correlation. The risk of charging too many EVs from a single distribution transformer depends on the assumed transformer size, number of EVs considered [15, 16], charging levels, and the degree of correlation between neighbors charging choices, such as charge power level and starting time. 


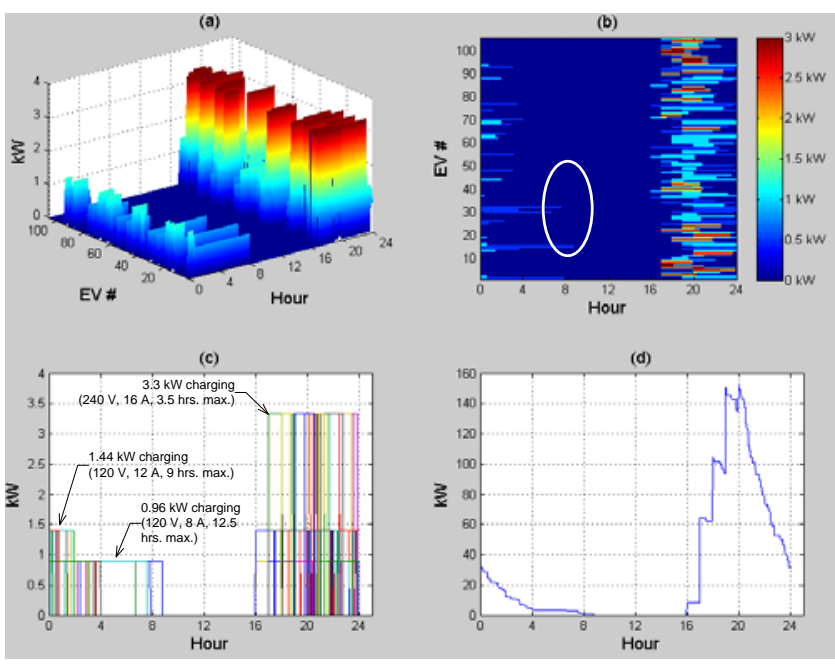

Fig. 5. Charging profiles for all 100 electric vehicles in a 24-hour period. Data interval: $1 \mathrm{~min}$.

\section{Transformers}

Utilities have concerns about the fast growth and impact of highly concentrated PV systems and EVs. These concerns stem from PV systems output power dependency on weather conditions and from uncontrolled distributed generation growth and placement, both in terms of electric phase and geographical distribution. Utility concern also stems from asset management of all grid components, which in this work, is limited to the operational state of the distribution transformers as a way to demonstrate the study approach. This concern has led to further uncertainty, since transformers were already operating at capacity before PV systems and EVs were deployed. The following analysis estimates the real power flow experienced by said transformers and reports their estimated operating conditions.

\section{1) Transformer Net Power Flow}

Following the conventions used in previous figures, Fig. 6 shows the net real-power flow through each of the 94 distribution transformers.

a) Figure 6 (a) shows the net power flow through each transformer, measured looking into the primary-side terminals (in the forward or step-down direction). The transformers are enumerated 1 through 94 in accordance with Tables II through IV. The corresponding color map is on the right side of the figure. As observed from the larger lower circled area, many transformers experience diurnal reverse (negative) real power flows of $-12 \mathrm{~kW}$ due to higher PV-to-load ratios. Power reversal depends on the time of day, weather conditions, and how many residents have unused PV capacity. Similarly, the smaller circled area shows transformers experiencing peak loads of $\sim 40 \mathrm{~kW}$ in the evening hours. This view, similar to Fig. 3 (a), helps finding similar behavior in transformers by noticing peaks and valleys on the surface.

b) Figure 6 (b) highlights times when transformers experience reverse active power flow. According to the darker blue regions (vertical circle), reverse flows occur on many transformers between 8-4 PM. These flows are aligned with
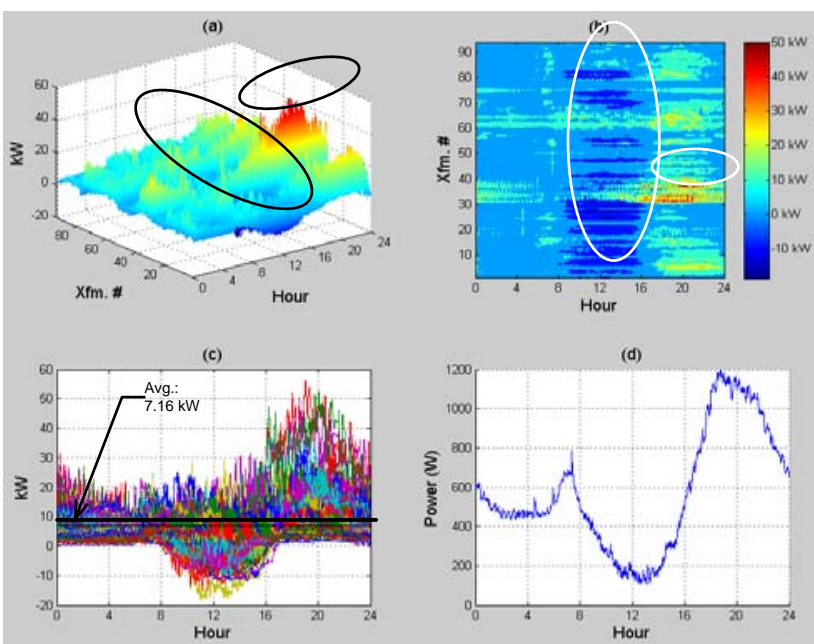

Fig. 6. Real power through all distribution transformers.

diurnal PV output, but vary by transformer. The horizontal circle shows some transformers loaded at $\sim 40 \mathrm{~kW}$. The dominant color on this contour plot is cyan, which suggests that the typical transformer usage at the Mueller community is $\sim 7 \mathrm{~kW}$.

c) Figure 6 (c) shows the load profiles for all transformers, which include residential load, PV generation, and EV charging. Clearly from this view, several transformers now act as 240-7.2 kV step-up transformers due to excess PV generation. This excess real power flows back into the grid but is consumed by neighboring loads on the same electrical phase.

d) Figure 6 (d) demonstrates aggregated transformer load; that is, the load-sum of all 94 distribution transformers, as seen from the lateral service entrance. This load includes the residential load, PV generation, and EV charging through all transformers. As expected, peak consumption occurs during breakfast and dinner time. Interestingly, these peaks are timeseparated by a low daytime power demand. This low demand is a direct result of having a high concentration of grid-tied PV systems producing only real power, and all behind the same service lateral.

\section{2) Transformer Percent Utilization}

Although Fig. 6 shows the direction of real power flow in each transformer, it does not convey transformer-utilization information. Consider Fig. 7, which shows utilization as a percentage of the total rated capacity measured in VA for each transformer. The four views in Fig. 7 indicate the following:

a) Figure 7 (a) reveals that some transformers are about $90 \%$ utilized. These few transformers, however, do not experience sustained loads at these levels. Instead, this loading condition is intermittent.

b) Figure 7 (b) shows the duration of the high-load conditions, which also confirms that this loading is intermittent. The dominant color in this view is dark blue, which indicates that most of the time the transformers operate between $10-30 \%$ of their rated capacity. 


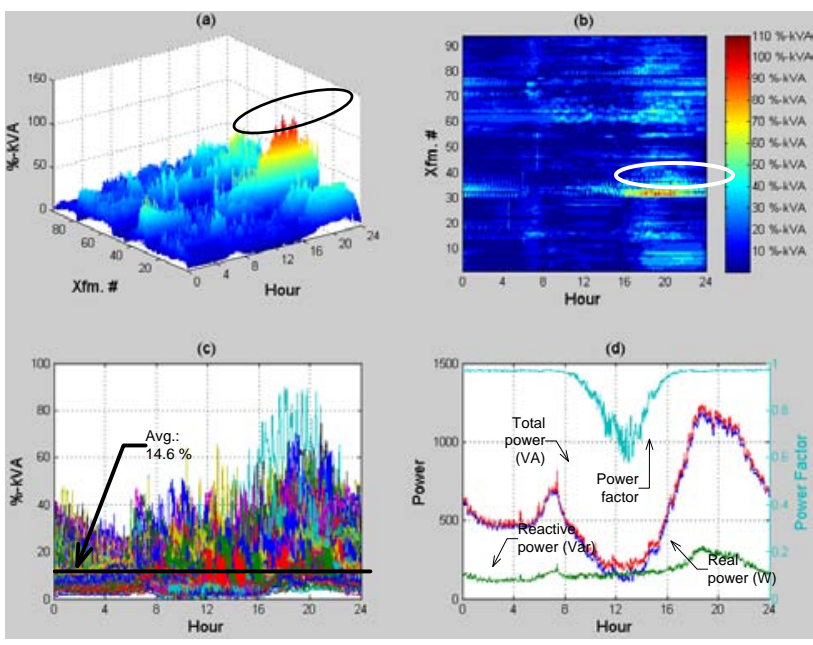

Fig. 7. Percent-utilization of all distribution transformers.

c) Figure 7 (c) authenticates the load profiles (VA) of all transformers. The average transformer consumption over all transformers over the 24-hour period analyzed in this work is $14.6 \%$. This percent-utilization is consistent with the surface level in Fig. 7 (a) and dominant color in Fig. 7 (b). Additionally, this view also shows little correlation among transformers load. It should be noted that this view is different from Fig. 6 (c); transformer utilization, in \%-VA, is always positive.

d) Figure 7 (d) shows aggregated transformer throughput (W, Var, and VA) as seen from the lateral service entrance. This power throughput includes both core and conduction losses, residential load, PV generation, and EV charging. This figure also shows a trace for the power factor against the right-side axis. The power factor trace shows poor daytime power conditions due to excess grid-tied PV generation in this community. It should be acknowledged that this poor power factor condition is not a byproduct of high reactive load. However, it is a byproduct of a significant decrease in real power demand through the lateral and into the neighborhood because of the real power generated within the neighborhood by grid-tied PV systems. Between $11 \mathrm{AM}$ and $2 \mathrm{PM}$, the utility supplies nearly the same amount of real power as it does reactive power. During these times, the utility experiences power-factor conditions as low as $\sim 0.6$. This power factor condition, however, is only observed from the lateral service entrance and does not represent the power factor seen from the substation.

\section{3) Transformer Voltage Profiles}

Figure 8 shows the secondary-side voltage profiles of all transformers using two views. The left side view shows the profiles over time and in per-unit, using 94 traces (one per transformer). As indicated from the daytime hours, PV systems cause voltage swells at the transformer above $1.0 \mathrm{pu}$ (on a $240 \mathrm{~V}$ base) for several transformers. However, this voltage swell is minor. Also, from the left-side view, EVs lower the transformer voltage during the evening hours. Both situations appear to be within the required service voltage (240 V) levels.

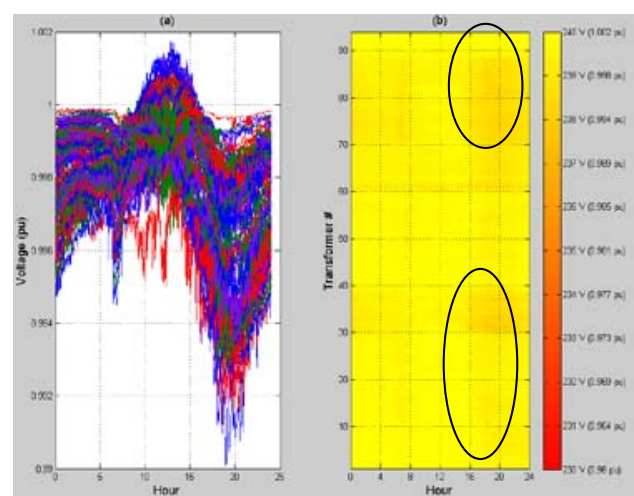

Fig. 8. Voltage profiles on transformer secondary sides

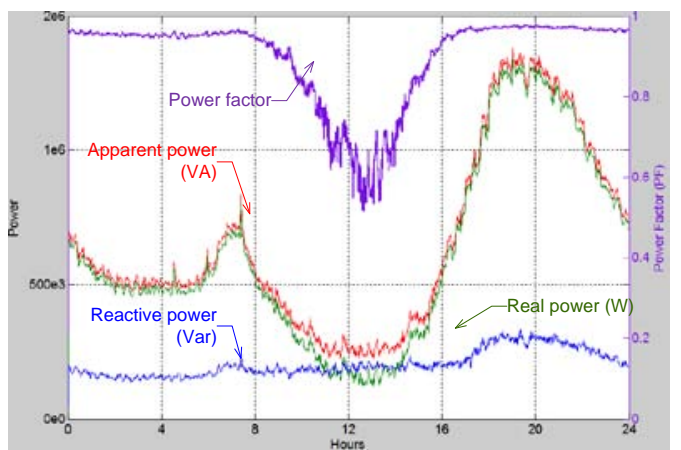

Fig. 9. Total power demand as seen from the lateral service entrance

The minimal impact of PV systems and EVs on distribution transformer voltage can be explained by referring to the transformer percent impedance ( $\mathrm{Z} \%$ ) nameplate values listed in Table I. The $\mathrm{Z} \%$ value approximates (in \%) the voltage drop across the transformer series impedance under full-load current [17]. As learned from Fig. 7 (c), most transformers do not operate at capacity (or full-load current); hence, their voltage drop is less than the nameplate $\mathrm{Z} \%$ value-even with the high concentrations of PV systems and EVs modeled herein.

The right side of Fig. 8 shows a filled 2-D contour representation of the same voltage profiles. The dominant color is yellow, which indicates that most transformers operate most of the time at values near $240 \mathrm{~V}$. The circled regions show times of the day when the transformer voltages are highest. Voltages greater than 1.0 p.u. are caused by PV systems producing localized power, which reduces the transformer's current and voltage drop. Similarly, in the afternoons, the filled contour plot exhibits darker regions corresponding to lower voltages due to EV charging.

\section{E. Lateral Power Demand}

Figure 9 shows the total power consumption of the community, including distribution losses in cables and transformers. The real power trace is larger than that in Fig. 7 (d) due to the inclusion of losses. As found during the diurnal hours, the power factor experienced by the utility at the lateral serving this community can be reduced to $\sim 0.6$ as real power is generated locally by the PV units in the neighborhood. Consistent with this observation, the reactive power is still provided by the utility. That is, residences and other grid 
components, such as transformers, still rely on the utility for reactive power although the utility does not bill for it. This has two implications: first, residences cannot become gridindependent unless they produce their own reactive power; second, the utility must schedule generation to provide this reactive power even though it is not billed to the customer.

Recently, Germany has implemented a standard that specify that the inverters on PV systems must supply both real and reactive power [18]. However, this requirement may lead to conflicts with implementation of IEEE Standard 1547 [8], which may suggest another potential limitation of this standard, such as the impossibility of powering loads during power outages - e.g. after natural disasters. Such a limitation [8] may merit its revision before the conventional timeframe beyond the changes already agreed within IEEE SCC21.

\section{CONCLUSIONS}

The use of recorded residential data as input for the system model is an approach that allows characterizing all transformers and, subsequently, identifying those under greatest stress. This approach improves the understanding of the distribution system behavior and is expected to be particularly beneficial in anticipating problems that could be caused by the additional acquisition of grid-tied PV systems and EVs. Typically, it is not cost-effective to upgrade functional distribution systems. This work demonstrates, however, that the additional information about the state of the distribution system provided by smart metering can be used to target improvements in response to customer choices and lead to cost-effective reliability enhancements for the distribution system.

It appears the distribution transformers at Mueller are sized to meet diverse EV charging levels in the evening hours. This observation is based on randomized charging levels (120 and $240 \mathrm{~V}$ ), duration, and plug-in time. There is also merit in the utility's foresight to deploy $50 \mathrm{kVA}$ transformers at this community in anticipation of the high concentrations of emerging technologies.

Key observations made from modeling of this particular system include:

- Most of the electrical footprint observed in this work stemmed from PV systems rather than from EVs. This footprint was manifested as reversed power flows in transformers, daytime voltage swells ( $>240 \mathrm{~V}$ ), poor power factor conditions through transformers and at the lateral, and reduced lateral power demand during day time.

- System imbalances can be induced from the uncontrollable placement of PV generation, preferentially on phases $a, b$, or c. While the installation of a PV system is the decision of the homeowner and not the utility, the modeling described here could help the utilities rebalance their systems prior to any problems caused from excessive voltage unbalance.

- Bidirectional power flow in networks designed for flow in one direction (from source to load) improves distribution efficiency, provides voltage support, and reduces utility-side generation demand; however, it also introduces protection and control complexities not present before.

The voltage profiles at the transformers are within acceptable levels. This result suggests that residents are not affected by the high concentration of PV systems and EVs at this community and at this time. It is also noted that customers are credited on their electric bill for $100 \%$ of the $\mathrm{kWh}$ produced by their solar system at a Value-of-Solar Factor (currently \$ 0.128 / kWh).

The visualization techniques presented in this work are somewhat unorthodox in power engineering. However, they also allow viewing the performance and uncorrelated behavior of all assets of a given type at once. Computer simulations producing such views can be a valuable tool in enhancing situational awareness.

An obvious extension of this work is to model an even larger system to show that using substation data, smart meter data, and a model of the system, one can predict, with a high degree of confidence, the operational state of all power apparatus downstream of a feeder. Installation of additional sensors in the distribution system is likely unnecessary. Loadend consumption and generation profiles appear to suffice.

\section{ACKNOWLEDGMENTS}

The authors thank Brewster McCracken, Bert Haskell, Scott Grantham, and Chris Holcomb from Pecan Street, Inc. for making available the data for analysis. In addition, the authors thank Charles Robinson, Sharon Bickford, and Kurt Stogdill from Austin Energy, and William Muston and David Woody from Oncor in Dallas, Texas for their guidance and providing power-utility engineering experience.

\section{REFERENCES}

[1] F. Katiraei and J. R. Aguero, "Solar PV Integration Challenges," Power \& Energy Magazine, pp. 62-71, May/June, 2011.

[2] J. R. Aguero, P. Chongfuangprinya, S. Shao, L. Xu, et al., "Integration of plug-in electric vehicles and distributed energy resources on power distribution systems," in IEEE Electric Vehicle Conference, 2012, pp. $1-7$.

[3] J. L. Calero Lagares, J. M. Roldan Fernandez, M. Burgos Payan, and J. M. Riquelme Santos, "Synergy of photovoltaic generators and Electric Vehicles in a low voltage distribution grid," in PowerTech, 2011 IEEE Trondheim, 19-23 June 2011, 2011, pp. 1-6.

[4] G. Kerber and R. Witzmann, "Loading Capacity of Standard Oil Transformers on Photovoltaic Load Profiles," in World Renewable Energy Congress (WRECX), 2008, pp. 1198-1203.

[5] J. C. Whitaker, AC power systems handbook, 3rd ed. Boca Raton, FL: CRC/Taylor \& Francis, 2007.

[6] H. Jimenez, H. Calleja, R. González, J. Huacuz, et al., "The impact of photovoltaic systems on distribution transformer: A case study," Energy Conversion and Management, vol. 47, pp. 311-321, 2005.

[7] A. Kwasinski, A. Toliyat, and F. M. Uriarte, "Effects of high penetration levels of residential photovoltaic generation: Observations from field data," in International Conference on Renewable Energy Research and Applications (ICRERA), Nagasaki, Japan, Nov. 11-14, 2012.

[8] IEEE Std 1547-2003 Interconnecting Distributed Resources with Electric Power Systems.

[9] A. Omole, "Voltage stability impact of grid-tied photovoltaic systems utilizing dynamic reactive power control," Dissertation, Departnent of Electrical Engineering, University of South Florida, 2010.

[10] P. Kundur, Power System Stability Analysis, 5th ed.: McGraw-Hill, 1994. 
[11] Y. Ruifeng and T. K. Saha, "Investigation of Voltage Stability for Residential Customers Due to High Photovoltaic Penetrations," Power Systems, IEEE Transactions on, vol. 27, pp. 651-662, 2012.

[12] C. Whitaker, J. Newmiller, M. Ropp, and B. Norris, "Renewable Systems Interconnection Study: Distributed Photovoltaic Systems Design and Technology Requirements," Available [online] http://www1.eere.energy.gov/solar/pdfs/distributed_pv_system_design.p df2008.

[13] G. J. Shirek and B. A. Lassiter, "Solar plant modeling impacts on distribution systems PV case study," in Rural Electric Power Conference (REPC), 2012 IEEE, 15-17 April 2012, 2012, pp. B5-1-B510.

[14] National Fire Protection Association, NFPA 70 - National Electric Code, 2005.

[15] S. Shao, M. Pipattanasomporn, and S. Rahman, "Grid integration of electric vehicles and demand response with customer choice," IEEE Trans. Smart Grid, vol. 3, pp. 543-550, 2011.

[16] S. Shao, M. Pipattanasomporn, and S. Rahman, "Demand response as a load shaping tool in an Intelligent grid with electric vehicles," IEEE Trans. Smart Grid, vol. 2, pp. 624-631, 2011.

[17] J. H. Harlow (editor), Electric Power Transformer Engineering: CRC Press, LLC, 2004.

[18] W. Yan, M. Braun, J. von Appen, E. Kämpf, et al., "Operation Strategies in Distribution Systems with High Level PV Penetration," in Proc. ISES Solar World Congress 2011, 2011.

\section{BIOGRAPHIES}

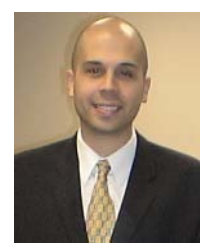

Fabian Marcel Uriarte, $\mathbf{P h D}$ is a Research Associate at the Center for Electromechanics at the University of Texas at Austin. He obtained his BS and MS from Virginia Tech, and $\mathrm{PhD}$ from Texas A\&M University-all in electrical engineering. He is power system modeling and simulation specialist and is currently the technical lead behind modeling the Smart Grid at the Mueller Community.

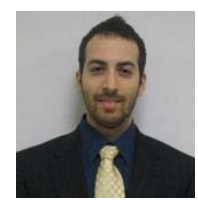

Amir Toliyat Amir Toliyat is a research assistant and $\mathrm{PhD}$ student in electrical engineering at The University of Texas at Austin (UT). He received the BS and MS degrees from Texas A\&M University and UT, respectively. His research interests include distributed generation and smart grids.

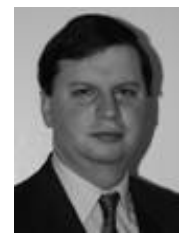

Alexis Kwasinski, $\mathbf{P h D}$ is an Assistant Professor in the Department of Electrical and Computer Engineering at the University of Texas at Austin. He obtained his MS and PhD in electrical engineering from the University of Illinois at Urbana-Champaign. His past industry experience includes working for Telefónica of Argentina and for Lucent Technologies Power Systems. Dr. Kwasinski is an Associate Editor for the IEEE Transactions on Energy Conversion.

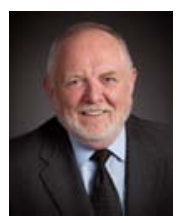

Robert E. Hebner, PhD (S'70-M'71-SM'83-F'93) is Director of the Center for Electromechanics at the University of Texas at Austin. The Center develops technology, primarily for novel motors, generators, and suspension components, and teams with companies to get the technology into the market. Previously, Dr. Hebner was the acting Technology (NIST). Director of the U.S. National Institute of Standards and 